\title{
Search for photospheric footpoints of quiet Sun transition region loops
}

\author{
J. Sánchez Almeida ${ }^{1}$, L. Teriaca ${ }^{2}$, P. Sütterlin ${ }^{3}$, D. Spadaro ${ }^{4}$, U. Schühle ${ }^{2}$, and R. J. Rutten ${ }^{3}$ \\ ${ }^{1}$ Instituto de Astrofísica de Canarias, 38205 La Laguna, Tenerife, Spain \\ e-mail: jos@iac.es \\ 2 Max-Planck-Institut für Sonnensystemforschung, Max-Plank Str. 2, 37191 Katlenburg-Lindau, Germany \\ e-mail: [teriaca; schuehle]@mps.mpg.de \\ 3 Sterrenkundig Instituut, Universiteit Utrecht, PO Box 80000, 3508 TA Utrecht, The Netherlands \\ e-mail: [P.Suetterlin;R.J.Rutten]@astro.uu.nl \\ 4 INAF-Osservatorio Astrofisico di Catania, 95123 Catania, Italy \\ e-mail: dspadaro@oact.inaf.it
}

Received 20 June 2007 / Accepted 18 September 2007

\section{ABSTRACT}

\begin{abstract}
Context. The footpoints of quiet Sun Transition Region (TR) loops do not seem to coincide with the photospheric magnetic structures appearing in traditional low-sensitivity magnetograms.

Aims. We look for the so-far unidentified photospheric footpoints of TR loops using G-band bright points (BPs) as proxies for photospheric magnetic field concentrations.

Methods. We compare TR measurements with SoHO/SUMER and photospheric magnetic field observations obtained with the Dutch Open Telescope.

Results. Photospheric BPs are associated with bright TR structures, but they seem to avoid the brightest parts of the structure. BPs appear in regions that are globally redshifted, but they avoid extreme velocities. TR explosive events are not clearly associated with BPs.

Conclusions. The observations are not inconsistent with the BPs being footpoints of TR loops, although we have not succeeded to uniquely identify particular BPs with specific TR loops.
\end{abstract}

Key words. Sun: activity - Sun: magnetic fields - Sun: photosphere - Sun: transition region

\section{Rationale}

The solar Transition Region (TR) is defined as the part of the solar atmosphere characterized by temperatures from $2 \times 10^{4} \mathrm{~K}$ (upper chromosphere) to $10^{6} \mathrm{~K}$ (corona), and densities from $\sim 10^{10} \mathrm{~cm}^{-3}$ to $\sim 10^{8} \mathrm{~cm}^{-3}$ (e.g., Mariska 1992). In classical 1-D coronal models (e.g., Gabriel 1976), the TR is a thin $(\sim 100 \mathrm{~km})$ thermal interface between the cooler chromosphere and the hotter corona. Although such interface regions must exist at the footpoints of large active region coronal loops, they appear to be responsible of only a small fraction of the TR emission (e.g., Athay 1982; Feldman 1983). The quiet Sun TR, in particular, does not represent a continuous transition between the chromosphere and the corona. Rather, sensitive UV observations show the upper solar atmosphere to consist of a hierarchy of loop structures with different temperatures and extents (e.g., Dowdy et al. 1986; Feldman et al. 2000; Feldman 2002). Small cool looplike structures fill most of the quiet Sun images and spectroheliograms obtained in lines formed at TR temperatures (see Feldman et al. 1999, Fig. 7). The footpoints of such loops do not seem to be associated with known traditional magnetic structures. They lie across network boundaries with the footpoints presumably in the interior of supergranulation cells. Feldman et al. (2001) find no chromospheric counterpart near the apparent footpoints of the structures. Warren \& Winebarger (2000) find that the loops do not connect magnetic structures in full-disk magnetograms obtained with the Michelson Doppler Imager (MDI) aboard SoHO. This kind of magnetogram, however, does not have enough spatial resolution and sensitivity to reveal magnetic structures in supergranulation cell interiors. In fact, it has been known for a long time that such structures do exist (Livingston \& Harvey 1975; Smithson 1975). They show up as weak Hanle depolarization signals (e.g., Stenflo 1982; Faurobert-Scholl 1993; Trujillo Bueno et al. 2004), weak Zeeman polarization signals (e.g., Wang et al. 1995; Lin \& Rimmele 1999; Sánchez Almeida \& Lites 2000; Domínguez Cerdeña et al. 2003), and small bright points in intergranular lanes (Sánchez Almeida et al. 2004; de Wijn et al. 2005). According to numerical simulations (e.g., Cattaneo 1999; Vögler et al. 2005; Vögler \& Schüssler 2007; Stein \& Nordlund 2006) and observations (e.g., Sánchez Almeida \& Lites 2000; Sánchez Almeida et al. 2003; Domínguez Cerdeña et al. 2006), a complex magnetic field pervades the seemingly non-magnetic quiet photosphere. Theoretical arguments suggest that a significant part of such photospheric magnetic field actually reaches the TR and the corona (Schrijver \& Title 2003; Jendersie \& Peter 2006). Obviously, these ubiquitous magnetic fields seem to be the natural candidates for the so-far unidentified quiet Sun TR loop footpoints. If such conjecture turns out to be correct, it offers a new standpoint for studying and understanding the nature of the TR and its loops. In addition, it would provide a new 


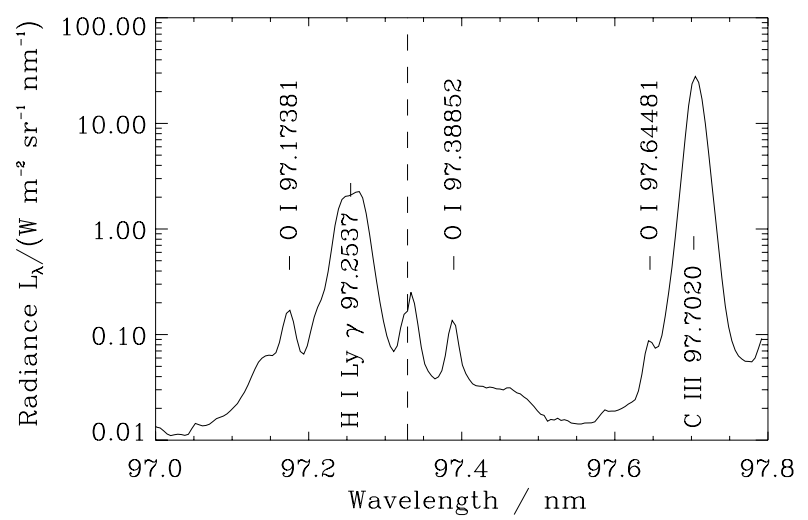

Fig. 1. Spectrum of the SUMER observations averaged over the full FOV. Spectral radiances are given in $\mathrm{W} \mathrm{m}^{-2} \mathrm{~s}^{-1} \mathrm{sr}^{-1} \mathrm{~nm}^{-1}$. All relevant lines are indicated with the known rest wavelengths (O I from Kelly 1987; C III and H I Ly $\gamma$ from Morton 2003). The vertical dashed line marks the boundary between the $\mathrm{KBr}$-coated and the bare sections of the SUMER detector.

scientific rationale for studying the magnetism of the quiet Sun. Guided by these ideas, we undertook a first exploratory study to identify the photospheric footpoints of the quiet Sun TR loops. Such work is described in the present paper.

The study requires simultaneous observations of the quiet Sun TR and the photospheric magnetic fields. The Solar Ultraviolet Measurements of Emitted Radiation (SUMER) spectrometer (Wilhelm et al. 1995) aboard SoHO was used to record the $97 \mathrm{~nm}$ to $98 \mathrm{~nm}$ spectral range (Fig. 1) that includes the H I Ly $\gamma 97.2 \mathrm{~nm}$ line $\left(T \sim 1.5 \times 10^{4} \mathrm{~K}\right)$ and the C III $97.7 \mathrm{~nm}$ line $\left(T \sim 8 \times 10^{4} \mathrm{~K}\right)$. The latter is one of the brightest lines of the solar VUV spectrum, allowing low-noise spectra to be recorded with exposure times of a few seconds. Being formed in the middle TR, the C III $97.7 \mathrm{~nm}$ line is an ideal tracer of the TR looplike structures (Feldman et al. 1999). As proxy for the quiet Sun magnetic fields, we employ high spatial resolution images in the so-called $\mathrm{G}$ band (the $\mathrm{CH}$ molecular band at $430 \mathrm{~nm}$ ). G-band bright points (BPs) in intergranular lanes are proxies for intense $\mathrm{kG}$ magnetic concentrations (Muller \& Roudier 1984; Berger et al. 1995, 1998). The reasons for using G-band BPs as magnetic tracers is twofold. Magnetic structures having $\mathrm{kG}$ fields represent only a very small fraction of the quiet Sun magnetic structures, which have field strengths in the full range from $0 \mathrm{kG}$ to $2 \mathrm{kG}$ (Sánchez Almeida \& Lites 2000; Socas-Navarro \& Sánchez Almeida 2002). However, the magnetic loops rooted in $\mathrm{kG}$ concentrations are expected to tipover higher-up (Domínguez Cerdeña et al. 2006), and so they have the largest probability of being the footpoints we are seeking. The second reason has to do with feasibility - unpolarized imaging is simpler than spectro-polarimetry, and G-band BPs are easy to detect provided that the imaging has enough spatial resolution (Title \& Berger 1996). We employ the $45 \mathrm{~cm}$ Dutch Open Telescope (DOT) which, using speckle image reconstruction techniques, can provide time series of diffraction limited images in a routine fashion (Rutten et al. 2004).

The paper is organized as follows. The observations, reduction, and alignment are described in Sect. 2. Aligning the ground-based images with the satellite images is critical, which justifies the details given in Sect. 2. The observational results are put forward in Sect. 3. The implications of such results are analyzed in Sect. 4.

\section{Observations, data reduction, and co-alignment}

The observations were carried out on March 25, 2006, from 9:00 UTC to 11:00 UTC. Due to poor weather conditions at the DOT site, it turned out to be the only useful time slot out of three attempts in a coordinated campaign involving SUMER, MDI (Scherrer et al. 1995), and DOT. This section describes the data sets plus the procedure that allowed us to bring the satellite images and the ground-based images to a common reference system.

\subsection{SUMER spectra and MDI magnetograms}

The SUMER instrument is a slit spectrometer and, therefore, images are obtained by raster scanning across the region of interest. Our data consist of six rasters taken sequentially and forming three pairs. Due to a failure of the SUMER A detector, only a strip of about $20^{\prime \prime}$ along the slit could be imaged and, therefore, a second raster scan was placed $25^{\prime \prime}$ towards the south of the previous raster. The same region near the center of the solar disk was observed three times - always in east-west direction - yielding the six scans mentioned above. Each single raster is made of 99 step positions taken with a cadence of $13 \mathrm{~s}$ (12 s exposure) and therefore lasting about $22 \mathrm{~min}$. Between rasters the solar rotation was compensated automatically by displacement of the field-of-view (FOV) towards west. One pair of rasters renders a FOV of about $100^{\prime \prime} \times 45^{\prime \prime}$ (see Fig. 2). After accounting for solar rotation, the step size of the scan turns out to be 1.'092, which is similar to the sampling interval in the direction along the slit (1.'015). SUMER has a spatial resolution of 1.5" (Lemaire et al. 1997), corresponding to about $1000 \mathrm{~km}$ on the Sun at the distance from SoHO to the Sun.

The spectra were corrected for flat-field inhomogeneities and for the geometrical distortion induced by the electronic readout scheme by using the standard routines available to SUMER users. We corrected for residual geometrical distortion in the spectral direction, that is significant at the edges of the detector. This was done by straightening the averaged (over the entire dataset) slit spectrum around the $\mathrm{O}$ I lines present in the observed spectral range (see Fig. 1). Lines from neutrals and singleionized species are known to show very small average Doppler shifts and can be used to obtain a wavelength calibration (e.g., Teriaca et al. 1999, and references therein). Finally, the data were calibrated to yield spectral radiances in $\mathrm{W} \mathrm{m}^{-2} \mathrm{sr}^{-1} \mathrm{~nm}^{-1}$. The recorded spectral range is shown in Fig. 1. The spectra were used to compute integrated radiances at selected bandpasses together with Doppler velocities, and line widths. Three bandpasses are mentioned in the paper: C III $97.7 \mathrm{~nm}(97.65-$ $97.75 \mathrm{~nm})$, pseudo-continuum (97.4-97.6 nm), and H I Ly $\gamma$ $(97.2-97.3 \mathrm{~nm})$. In order to obtain velocities and line widths, the line profiles of C III $97.7 \mathrm{~nm}$ and the underlying continuum were fitted with a single Gaussian plus a second-order polynomial. The procedure provides errors for the velocities and Doppler widths. The mean errors considering the full FOV turn out to be $1.5 \mathrm{~km} \mathrm{~s}^{-1}$ and $0.5 \mathrm{pm}$, respectively. When a rest wavelength of $97.702 \mathrm{~nm}$ is used (Morton 2003), the average shift over the entire dataset is $11 \mathrm{~km} \mathrm{~s}^{-1}$. This value is comparable to the values found in the literature for lines formed at similar temperatures (see e.g., Teriaca et al. 1999, and references therein). The structures appearing in the SUMER C III $97.7 \mathrm{~nm}$ maps have dimensions very similar to those described by Feldman et al. (1999, 2000) as cool loop-like structures clustering across the chromospheric network boundaries. The structures are less evident, but this is mostly due to our much smaller FOV, as compared to 


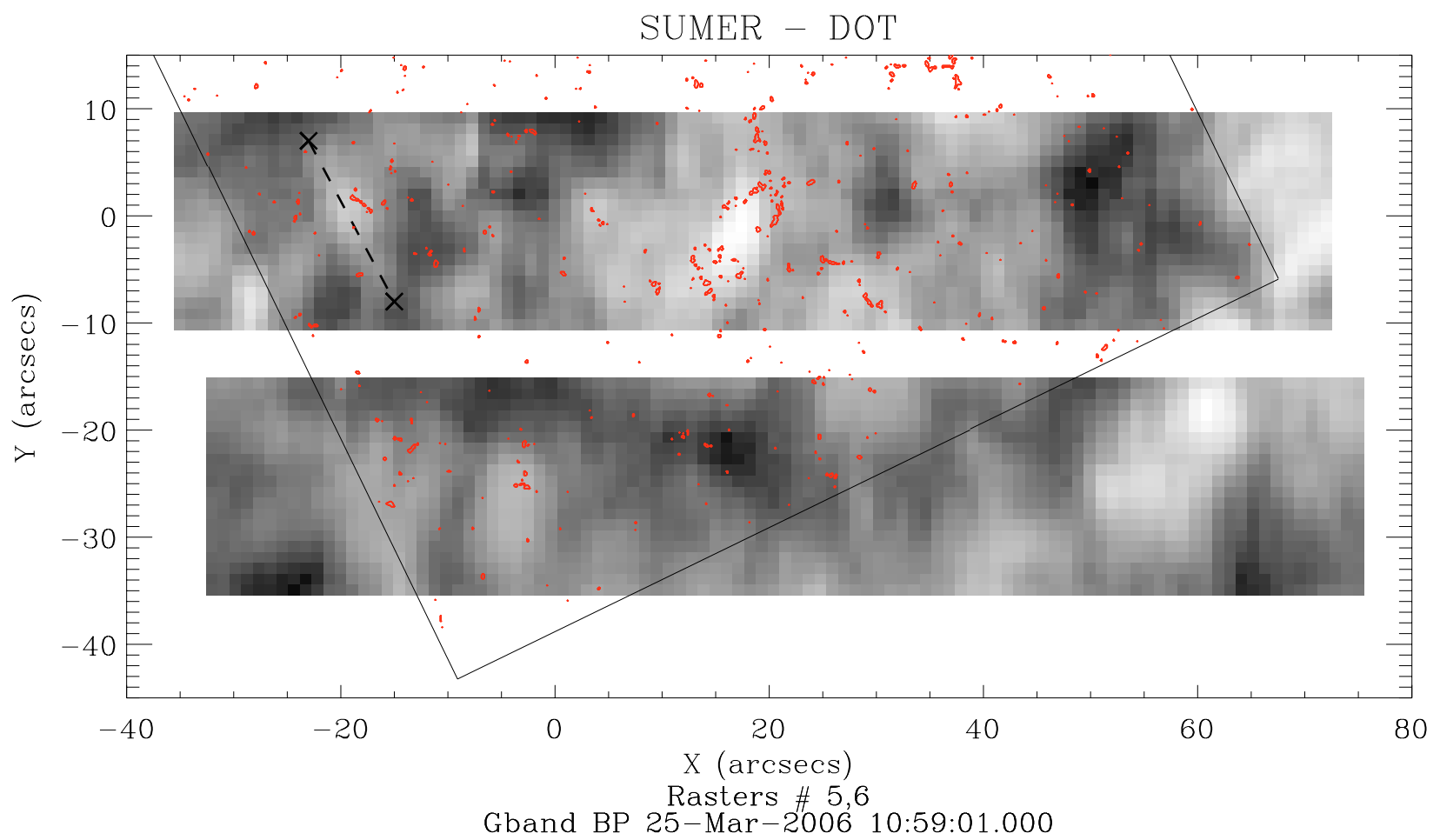

Fig. 2. SUMER C III $97.7 \mathrm{~nm}$ radiances of rasters 5 and 6 (the image) together with the position of the G-band BPs (the red contours). Coordinates are referred to the solar disk center as provided by the MDI magnetograms. The C III $97.7 \mathrm{~nm}$ radiances are shown in a logarithmic scale. The rotated box outlines the DOT FOV. The dashed line limited by times symbols illustrates the kind of elongated structure that may be ascribed to individual loops projected on the solar surface.

the images published by Feldman and coworkers $\left(270^{\prime \prime} \times 300^{\prime \prime}\right)$. This fact, together with the lack of an objective definition of C III 97.7 nm loop-like structure, makes it uncertain identifying individual loops in our maps. One can find features in Fig. 2 that may be ascribed to individual loops projected on the solar surface (see the example shown as a dashed line limited by two times symbols), but such identification is not free from ambiguity. Because of this reason, we will use the term elongated bright structure to describe the elongated patchy structures in Fig. 2, keeping in mind, however, that they should be identified with the structures termed loops by Feldman et al. (1999).

In addition to the SUMER data, we also use full disk SoHO/MDI magnetograms taken from 9:00 UTC to 11:30 UTC with a cadence of $1 \mathrm{~min}$. In this case the pixel is 1'.98 square, with the noise level corresponding to some $16 \mathrm{G}$ (Scherrer et al. 1995; Liu \& Norton 2001).

\subsection{DOT images}

This work employs only part of the series of images routinely provided by the DOT (Rutten et al. 2004), in particular, we analyze G-band images, and $\mathrm{Ca}$ II $\mathrm{H}$ line core images. They are restored using speckle techniques (Rutten et al. 2004) which, under good seeing conditions, render diffraction limited images ( $\sim 0^{\prime} 2$ in the $\mathrm{G}$ band). The final images have a sampling interval of 0.071 , with a FOV of $85^{\prime \prime} \times 70^{\prime \prime}$ (see Fig. 3 ). The time series has an irregular cadence equivalent to one image every two minutes. The best seeing occurs by the end of the time sequence and, therefore, it is associated with SUMER rasters 5 and 6 (Fried's parameter $8.5 \mathrm{~cm}$ ).

As expected, the G-band images are full of BPs in intergranular lanes (Fig. 3). Automatic identification of BPs is not trivial since a simple threshold criterion does not suffice. Sometimes granules are brighter than BPs clearly visible in the intergranular lanes (e.g., Bovelet \& Wiehr 2003). We applied a simple algorithm consisting of three steps: (1) construction of a smoothed version of the G-band image which is computed after removal of the brightest features in the image, (2) subtraction of the smoothed image from the original one to enhance the small bright features, and (3) selection of the bright features in the subtracted image, but only when they are localized in dark areas of the smoothed image. The first step, where the brightest features are removed, produces a smoothed version of the image which is not contaminated by the presence of BPs. The difference between the full image and this smoothed version enhances the contrast of the G-band BPs, and this high-contrast image is used to select the bright features existing in the dark intergranular lanes. The algorithm does a good job, in the sense that it agrees with the visual identification of the BPs. It is not perfect, and a few bright borders of granules are misidentified as BP, and some BPs are overlooked. However, the identification suffices for the exploratory analysis carried out in this paper. The use of a different algorithm would slightly modify the number of selected BPs. As we explain in Sect. 3, the trends that we obtain remain the same for all the three SUMER raster pairs, which correspond to different seeing conditions at the DOT site. Since seeing modifies the number of detectable BPs, and it does not change the trends, the details on how the BPs are detected do not seem to alter our results.

\subsection{Alignment}

The quiet Sun magnetic network is well defined in both standard magnetograms and Ca II line core filtergrams (e.g., Beckers 1977). Our method uses this property to align satellite data (SoHO/MDI magnetograms) with ground-based data (DOT 


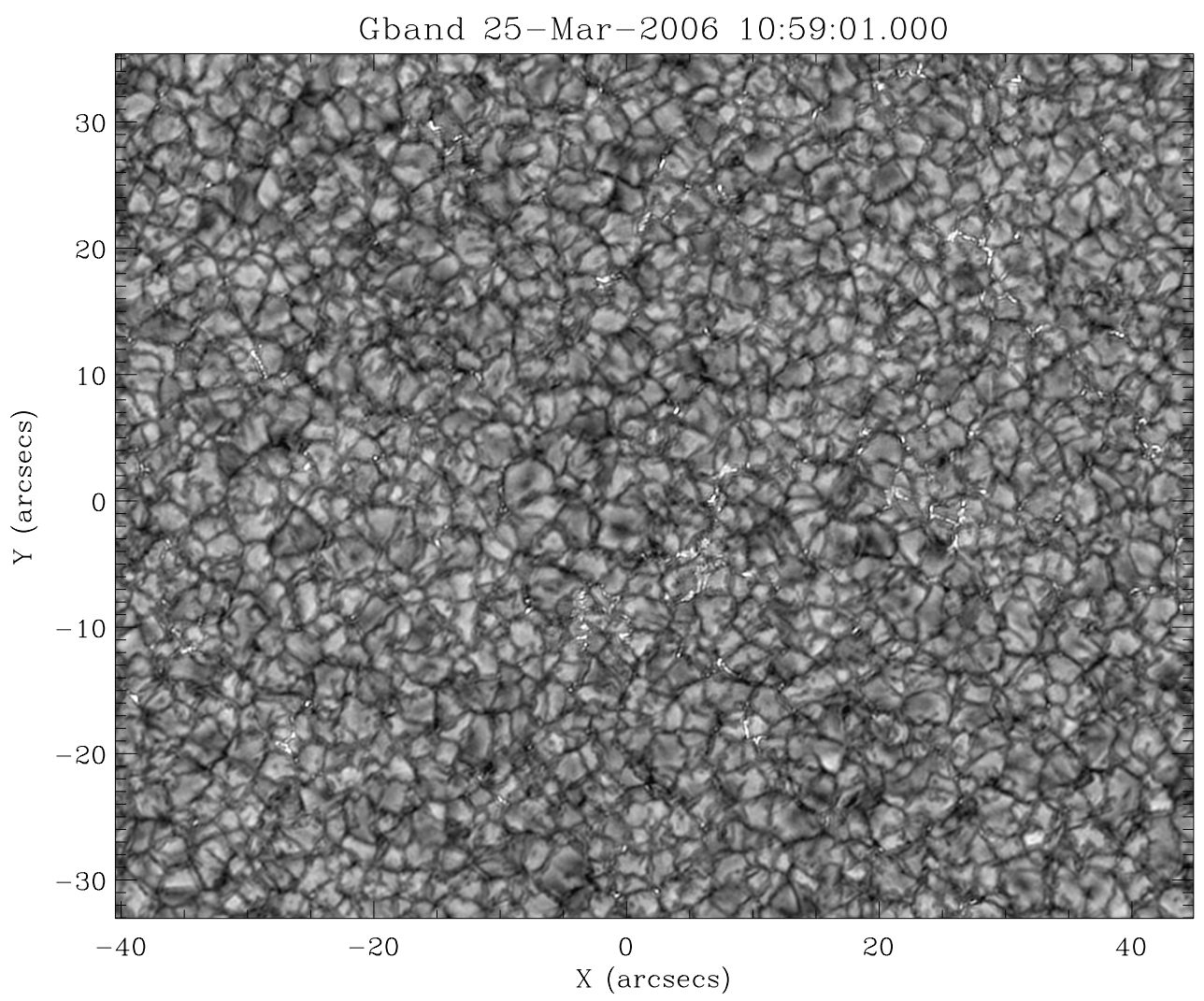

Fig. 3. Example of speckle reconstructed G-band image. It shows many G-band bright points tracing magnetic concentrations in intergranular lanes. The spatial coordinates are referred to the center of the image.

$\mathrm{Ca}$ II $\mathrm{H}$ images). We bring both SUMER raster scans and DOT G-band images to the spatial coordinates of MDI. Then SUMER raster scans and DOT G-band images can be superposed directly. The actual procedure is explained here in some detail.

Although SUMER data and MDI data come from a single satellite, they are not co-aligned. Errors in the raster mechanism and thermal excursions of the payload produce an unpredictable offset of up to $10^{\prime \prime}$. The SUMER to MDI co-alignment has been accomplished by comparing the pseudo-continuum SUMER image coming from the bandpass between $97.4 \mathrm{~nm}$ and $97.6 \mathrm{~nm}$ (Fig. 1), with the average among the MDI magnetograms taken during the time-span of the SUMER raster scan. The co-alignment is carried by trial and error, blinking on a computer screen the images of the SUMER rasters and the absolute value of the average MDI magnetogram. One of the images is then shifted with respect to the other to get the best match. The results are illustrated in Fig. 4. Repetitions of the exercise always yield the same offset within $\pm 1^{\prime \prime}$ (optimistic view), and $\pm 2^{\prime \prime}$ (conservative view). If the procedure uses Ly $\gamma$ photons instead of pseudo-continuum, then the alignment remains the same within the quoted uncertainties. We also tried aligning with and without removal of the solar rotation when averaging the magnetograms, and using a logarithm grayscale to represent SUMER radiances. No significant change is observed. The three couples of SUMER raster scans give the same offset.

The alignment between DOT and MDI is carried out by means of the $\mathrm{Ca}$ II $\mathrm{H}$ images. DOT images are both rotated and shifted with respect to SoHO images. The rotation is given by the angle between the geocentric North and the solar rotational North, which we set according to the ephemeris. As for the relative displacement, we use the same trial and error approach described above for the SUMER to MDI alignment. Since the resolution of the MDI magnetograms is much lower than the speckle restored DOT Ca II $\mathrm{H}$ images, the average among the burst of images gathered for speckle restoration is used for comparison. Errors are smaller than the SUMER to MDI alignment since the structures observed in DOT Ca II H and MDI are quite similar and therefore easy to identify. After repeating the trial and error process several times, one finds the displacements to be consistent within $1^{\prime \prime}$. DOT G-band images and DOT Ca II H are also misaligned. We find the shift between the images by cross-correlation. This method does not correct for the slight different orientation of the two images, and for a small difference of the spatial scales. However, the two effects leave a residual error always well below $1^{\prime \prime}$.

In short, the critical part of the alignment has been carried out by trial and error and, therefore, its uncertainty is difficult to estimate. However, judging the errors by the consistency of the trial and error process, they should be smaller than $2^{\prime \prime}$. This uncertainty is mostly set by the SUMER to MDI alignment, making all other errors negligible.

\section{Observational results}

The best DOT seeing occurred by the end of the time series, corresponding to SUMER rasters 5 and 6 . The number of G-band BPs in an image depends critically on the spatial resolution (Title \& Berger 1996; Sánchez Almeida et al. 2004), therefore, our analysis is focused on these last rasters and the best G-band image taken together with them. We also analyzed the other raster pairs, and other snapshots of the time series. The results are always consistent with those reported below. 


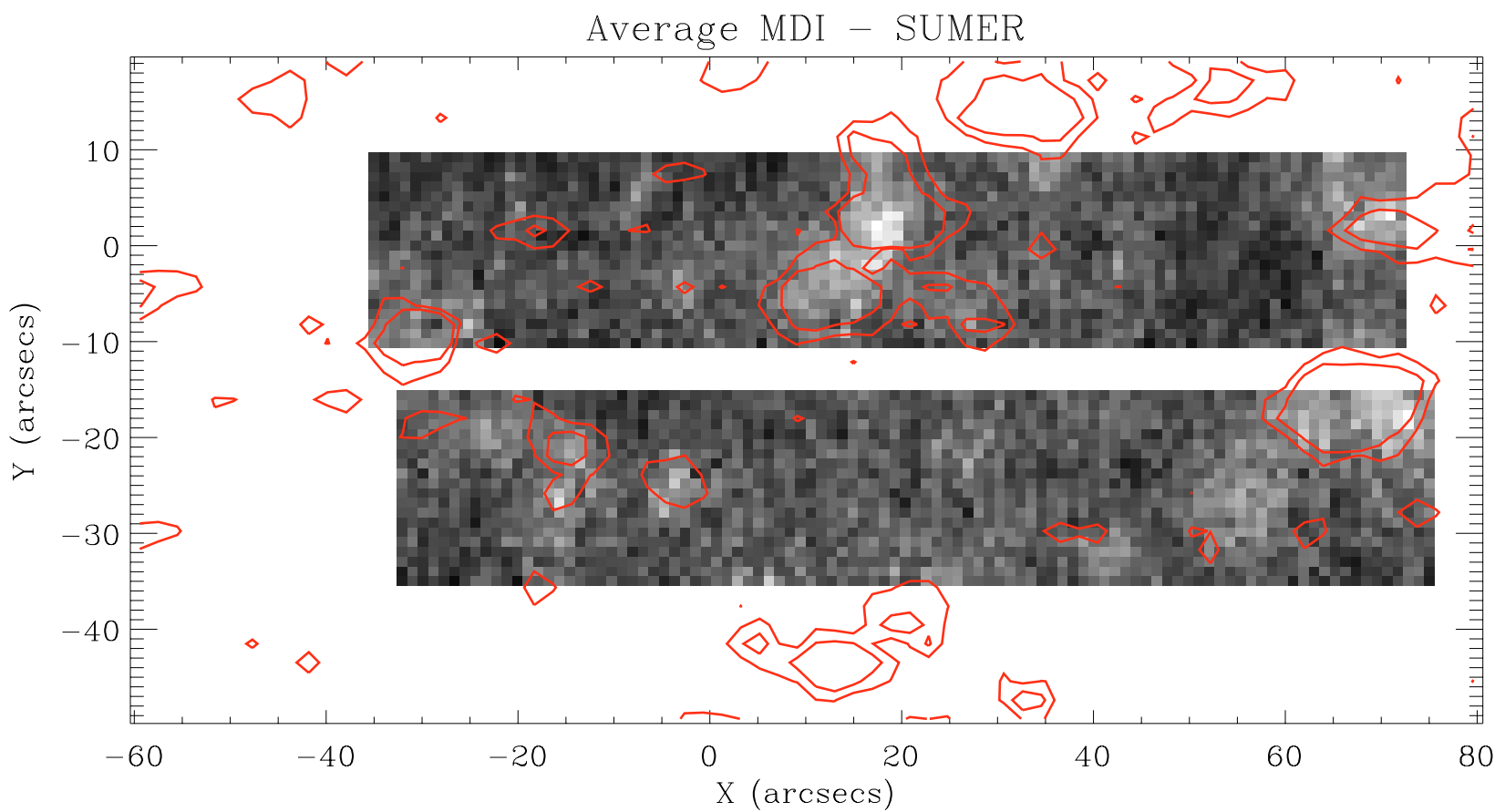

Fig. 4. Example of alignment between a SUMER pseudo-continuum image and the absolute value of the average MDI magnetogram. The latter is shown as a contour plot, with contours at $7 \mathrm{G}$ and $15 \mathrm{G}$. The spatial coordinates are referred to the solar disk center according to the MDI scale.

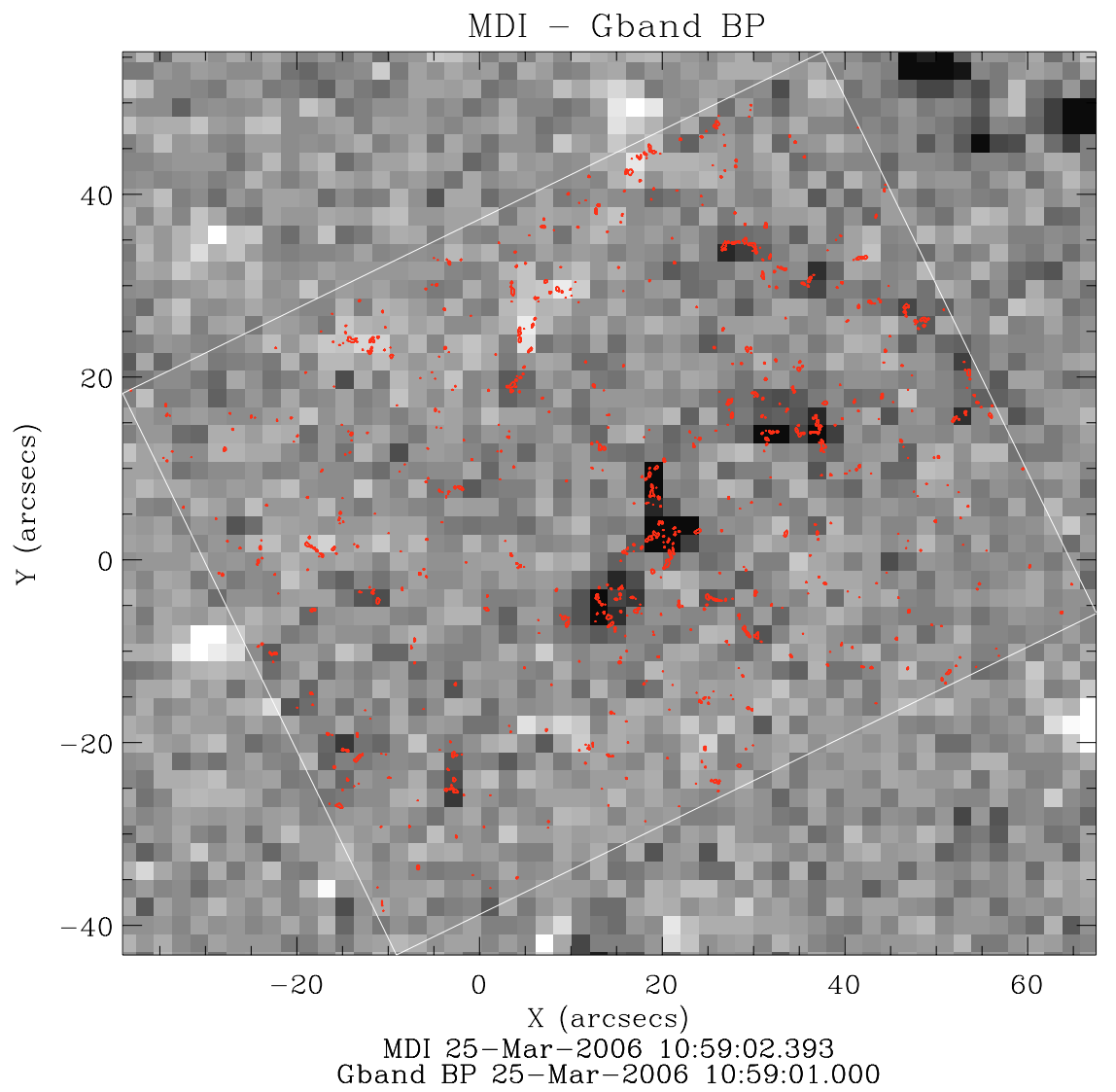

Fig. 5. MDI magnetogram (the image) together with the BPs existing in the G-band image (red contours). The magnetogram has been scaled from $-20 \mathrm{G}$ to $20 \mathrm{G}$, so that only the black and white pixels are well above the noise level ( $16 \mathrm{G})$. The coordinates are referred to the MDI solar disk center. The rectangle indicates the DOT G-band FOV. Note that these two images have not been aligned directly but via Ca II $\mathrm{H}$ images

\subsection{Radiances}

The G-band images show a significant number of BPs with no obvious counterpart in the MDI magnetograms (Fig. 5). As we point out in Sect. 1, a significant number of photospheric magnetic structures, and so of TR loop footpoint candidates, does not show up in traditional measurements. The G-band BPs existing 

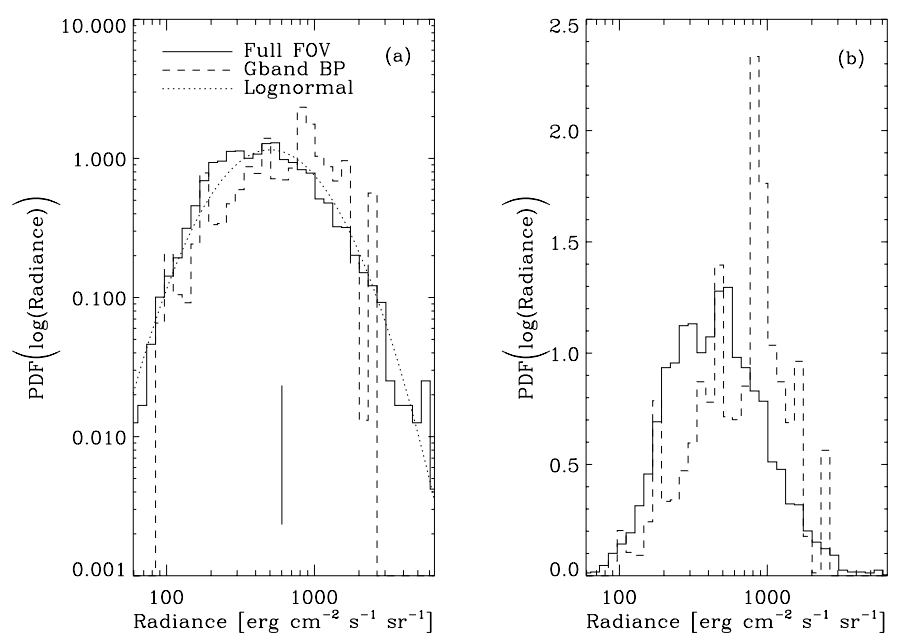

Fig. 6. a) Histogram (PDF) of the logarithm of the SUMER C III $97.7 \mathrm{~nm}$ radiance corresponding to the full SUMER FOV (the solid line), and at the pixels having G-band BPs (the dashed line). The dotted line corresponds to a lognormal approximation of the full FOV PDF. The vertical solid line indicates the mean radiance of the full FOV. b) Same as a) but showing the PDFs in a linear scale to emphasize the systematic increase of radiance in those pixels with BPs.

in the DOT image of best angular resolution (Fig. 3) are overplotted on the SUMER C III $97.7 \mathrm{~nm}$ image in Fig. 2. Visual inspection indicates that the photospheric BPs are associated with bright C III $97.7 \mathrm{~nm}$ features, although they seem to avoid the brightest cores. This is particularly clear for the case of the brightest C III $97.7 \mathrm{~nm}$ structure in the SUMER FOV, located at $X \simeq 15^{\prime \prime}$ and $Y \simeq 0^{\prime \prime}$ (see Fig. 2). In order to quantify this impression, we computed the histogram of C III $97.7 \mathrm{~nm}$ radiances for the full SUMER FOV, and for the C III $97.7 \mathrm{~nm}$ radiances at the positions of the G-band BPs (i.e., assigning the radiance of the closest SUMER pixel to each DOT pixel classified as G-band BP). The result is shown in Fig. 6, where the histograms have been normalized to one and termed PDF (acronym for probability density function). As expected (e.g. Pauluhn et al. 2000), the PDF of the full FOV is approximately lognormal (a parabola in a $\log -\log$ plot; the dotted line in Fig. 6a). The PDF of C III $97.7 \mathrm{~nm}$ radiances at the G-band BPs turns out to be shifted toward large radiances (see the dashed line). This shift is a feature common to all three pairs of raster scans. Note also the lack of very bright features in the G-band histogram - the dashed line in Fig. 6a drops down for radiances larger than $2500 \mathrm{~mW} \mathrm{~m}^{-2} \mathrm{sr}^{-1}$. This drop may unveil the tendency for the BPs to avoid the core of the bright C III $97.7 \mathrm{~nm}$ structures.

\subsection{Velocities and Doppler widths}

Figure 7 contains the map of velocities derived from C III $97.7 \mathrm{~nm}$ together with the G-band BPs. The bright and dark patches of the velocity image are devoid of BPs, which avoid extreme velocities. This view is corroborated by the histogram of velocities presented in Fig. 8. It shows the histograms (PDFs) for the full SUMER FOV (the solid lines), together with the histograms of velocities at the position of the G-band BPs (the dashed lines). The BP histogram lacks of the largest blueshifts and redshifts existing in the full FOV histogram. Another property easy to extract from Fig. $8 \mathrm{~b}$ is the global redshift of the SUMER spectra with BPs with respect to the full FOV velocities. The mean and standard deviation of the velocity distributions are $11.0 \pm 5.0 \mathrm{~km} \mathrm{~s}^{-1}$ (full FOV) and $12.9 \pm$ $5.3 \mathrm{~km} \mathrm{~s}^{-1}$ (BPs), which renders a relative shift of the order of $1.9 \mathrm{~km} \mathrm{~s}^{-1}$. The existence of such excess of redshift is a very robust result. The velocity histograms are made of hundreds of points, and they are well represented by Gaussians with widths of about $5 \mathrm{~km} \mathrm{~s}^{-1}$. The histograms of the means are necessarily much narrower, with widths of the order of $5 \mathrm{~km} \mathrm{~s}^{-1}$ divided by the square root of the number of individual velocities used to compute the means (e.g., Martin 1971). In our case this uncertainty of the means $\left(\leq 0.3 \mathrm{~km} \mathrm{~s}^{-1}\right)$ is much smaller than the separation between the means. Two final comments are in order. First, the difference of velocities cannot be caused by systematic errors biasing our wavelength calibration, since they would affect the two histograms in the same way. Second, similar shifts are present in all raster scans, reinforcing the results.

Nothing special seems to be associated with the maps of C III $97.7 \mathrm{~nm}$ widths except, perhaps, the fact that the largest widths do not coincide with BPs (see Fig. 9). The regions of largest C III $97.7 \mathrm{~nm}$ line width coincide with the largest blueshifts. One may naively think that these regions present systematic upflows of a few $\mathrm{km} \mathrm{s}^{-1}$, but they really contain much larger spatially unresolved upflows and downflows. The line widths are much larger than the line shifts, and a typical width of $0.02 \mathrm{~nm}$ corresponds to $60 \mathrm{~km} \mathrm{~s}^{-1}$ at C III $97.7 \mathrm{~nm}$.

\subsection{Explosive events}

Explosive Events (EEs) are believed to be the result of magnetic reconnection. They can be identified because the line profiles are strongly non-Gaussian, and they often coincide with locations of very large Doppler shifts and/or line widths derived by single-Gaussian fitting (Dere et al. 1989; Teriaca et al. 2004). We run a procedure to identify EEs by comparing the result of a single-Gaussian fit with the observed profiles. All profiles for which at least three contiguous spectral pixels consistently deviating by more than two sigma from the fit are flagged as EE. In our case we find 26 such spectra clustered in at least 7 patches. Unfortunately, most EE are outside the DOT FOV - the EEs selected in rasters 5 and 6 are represented in Fig. 10. From this very reduced statistics, we find no clear overlapping between EEs and G-band BPs, although each EE is not very far from a BP either. The spatial separation between BPs and EEs does not seem to be an artifact due to the lack of simultaneity between the SUMER spectra and the DOT image chosen to represent the photosphere. (Both EEs and BPs are transitory events lasting shorter than the SUMER scans. The DOT snapshot of best seeing may miss BPs existing during the individual EEs, masking a putative relationship between EEs and BPs.) BPs do not coincide with EEs even when the G-band image closest in time to each EE is used to search for BPs. Another possible bias has to do with the criterion to select EEs, which may overlook some of the weaker events or events that happen to produce a very broad but fairly Gaussian profile. However, as pointed out in Sect. 3.2, BPs also avoid regions of large Doppler widths or shifts, which reinforces the spatial disconnection between BPs and EEs.

\section{Discussion}

As far as we are aware of, this work represents the first attempt to find footpoints of quiet Sun TR loops in the interior of supergranulation cells. G-band bright points (BPs) are used as proxies for photospheric magnetic field concentrations which may anchor and guide the TR C III $97.7 \mathrm{~nm}$ structures simultaneously observed with SUMER. As we anticipated, most 


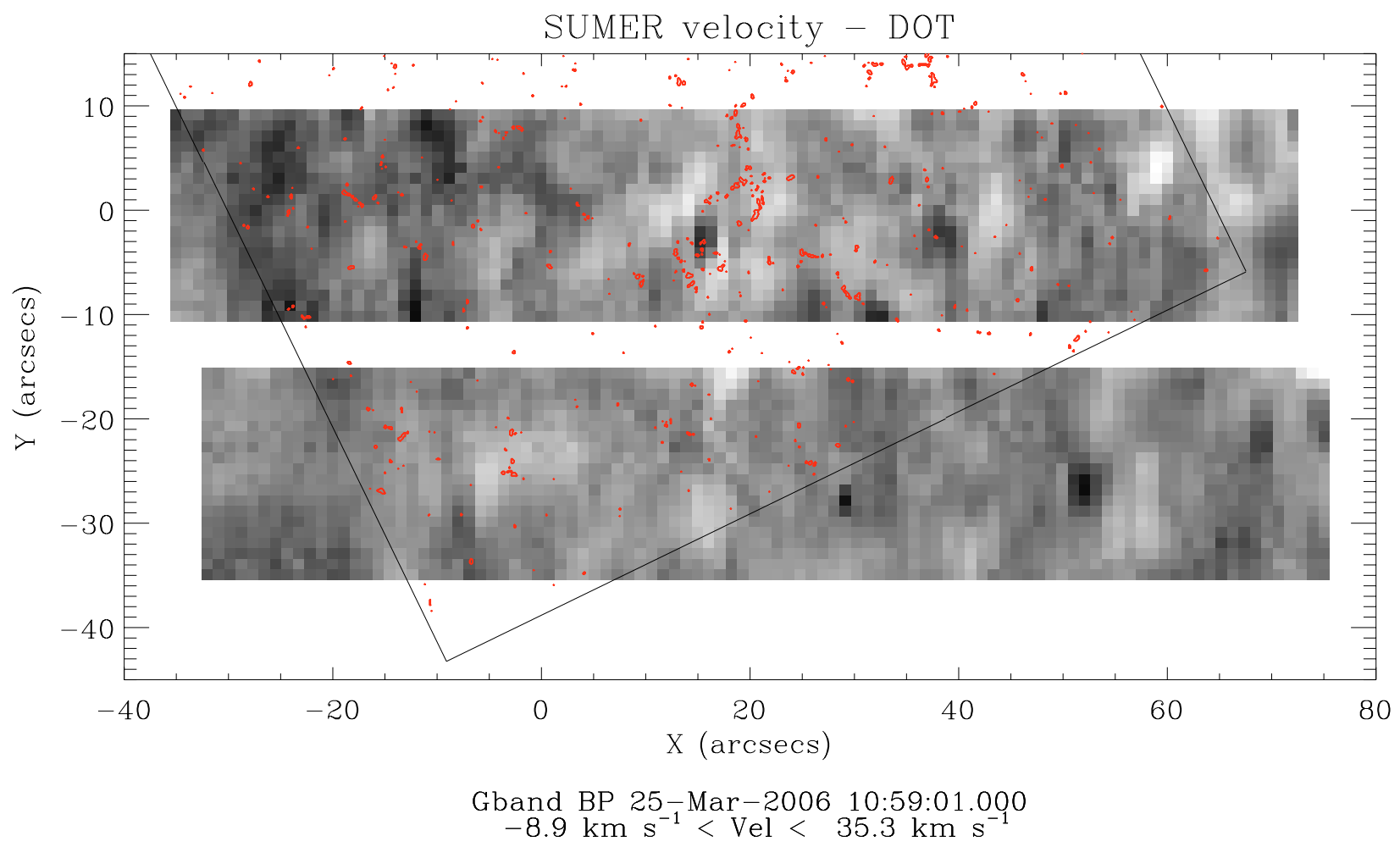

Fig. 7. C III $97.7 \mathrm{~nm}$ velocities (the image) together with the position of the G-band BPs (the red contours). The spatial coordinates and the box are the same as for Fig. 2. The image has been scaled between the minimum and maximum values indicated in the subtitle, with the white color representing the largest redshift.
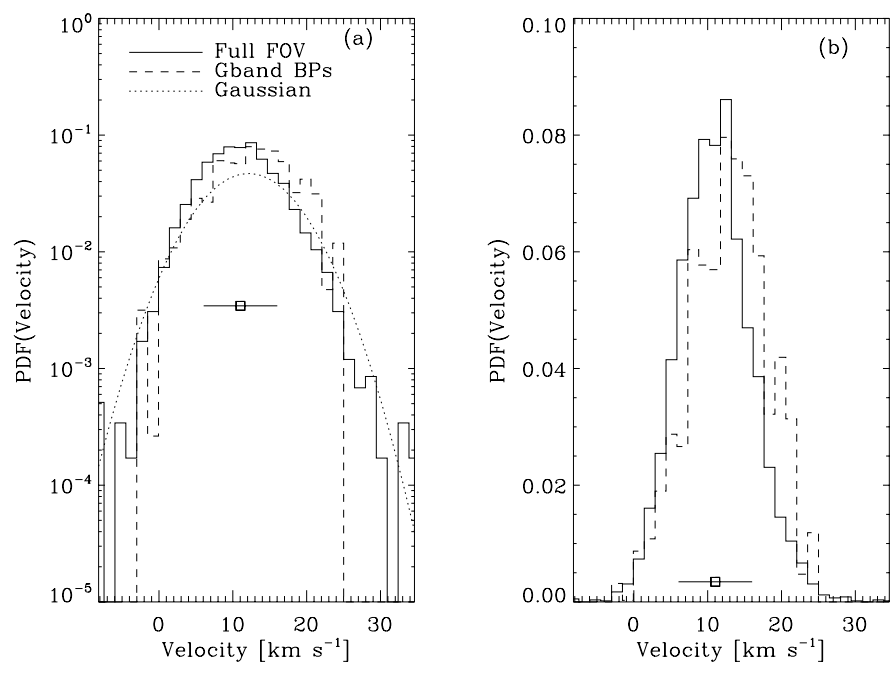

Fig. 8. a) Histogram (PDF) of the distribution of C III $97.7 \mathrm{~nm}$ velocities shown in a logarithm scale. As indicated by the inset, the solid line corresponds to the full SUMER FOV, whereas the dashed line shows the distribution of velocities in those places with G-band BPs. The dotted line corresponds to a Gaussian fit to the full FOV PDF. b) Same histograms as in a) but shown in a linear scale to emphasize the global redshift of the SUMER spectra in pixels with G-band BPs. Positive velocities correspond to redshifts. The square symbols with horizontal error bars represent the average plus-minus the standard deviation of the full FOV velocities.

photospheric magnetic concentrations do not appear in MDI magnetograms, therefore, traditional measurements miss TR loop footpoint candidates. Figure 2 presents our best effort to provide SUMER C III $97.7 \mathrm{~nm}$ radiances co-aligned with the underlying photospheric magnetic structures. Unfortunately, these and other similar maps do not allow us to uniquely identify Gband BPs with particular TR loops. Part of the difficulties have to do with identifying intrinsically fuzzy forms in our reduced FOV (Sect. 2.1). However, loosely using the term loop to denote the elongated structures present in the C III $97.7 \mathrm{~nm}$ maps, we find that some of them have BPs in the extremes, but some other BPs are not obviously associated with specific TR structures. What we find, however, is a series of new observational properties linking the BPs with the TR structures. In this sense the paper represents a study of the relationships between the TR observed in C III $97.7 \mathrm{~nm}$ and the presence of BPs. The observed properties are not inconsistent with the notion that BPs are indeed footpoints of TR loops, but to go beyond this conjecture is not free from speculation.

The observed G-band BPs are associated with bright C III $97.7 \mathrm{~nm}$ structures, although there may be a tendency for the BPs to avoid the brightest central parts of the TR structures (see Fig. 2 and the dashed line in Fig. 6b). In particular, the brightest structure in our FOV (Fig. $2, X \simeq 15^{\prime \prime}$ and $Y \simeq 0^{\prime \prime}$ ) is surrounded by two chains of BPs. It is tempting to think of the C III $97.7 \mathrm{~nm}$ structure as system of loops joining the two observed chains of BPs. However, this interpretation is inconsistent with the fact that the region containing the BPs and the C III $97.7 \mathrm{~nm}$ structure appears unipolar on the MDI magnetogram (see Fig. 5). A possible way to reconcile the existence of a system of closed loops with the MDI magnetogram is the presence of spatially unresolved mixed polarities, the MDI polarity being the dominating one. (The existence of unresolved mixed polarities in the quiet Sun is both, expected from numerical simulations, Cattaneo 1999, and observed, Sánchez Almeida \& Lites 2000.) Alternatively, the BPs surrounding the brightest C III $97.7 \mathrm{~nm}$ structure may be footpoints of a bunch of magnetic field lines forming a large multi-strand loop which closes 


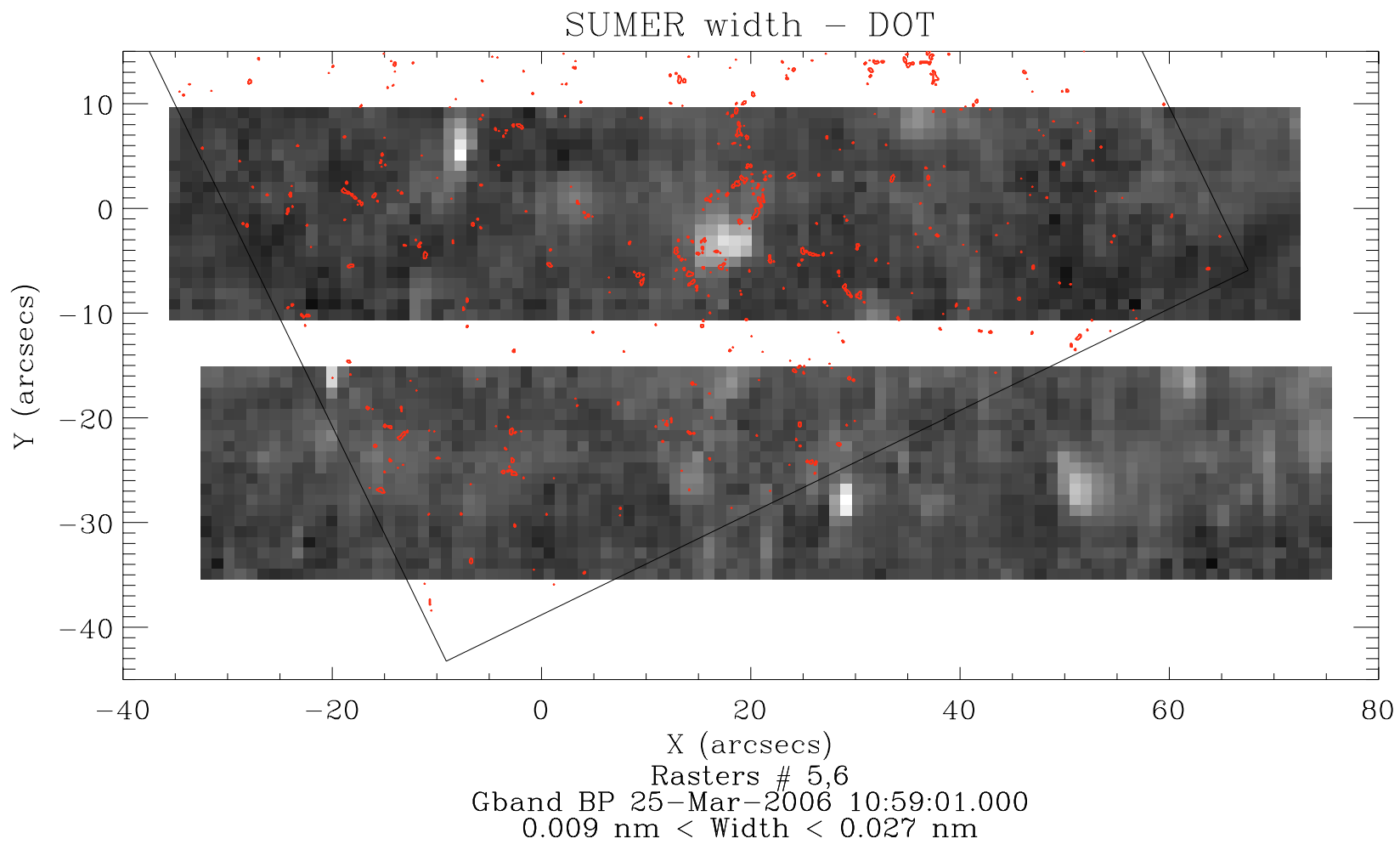

Fig. 9. C III $97.7 \mathrm{~nm}$ line widths in raster scans 5 and 6 (the image) together with the position of G-band BPs (the red contours). The spatial coordinates and the box are the same as for Fig. 2. The image has been scaled between the minimum and maximum widths indicated in the subtitle.

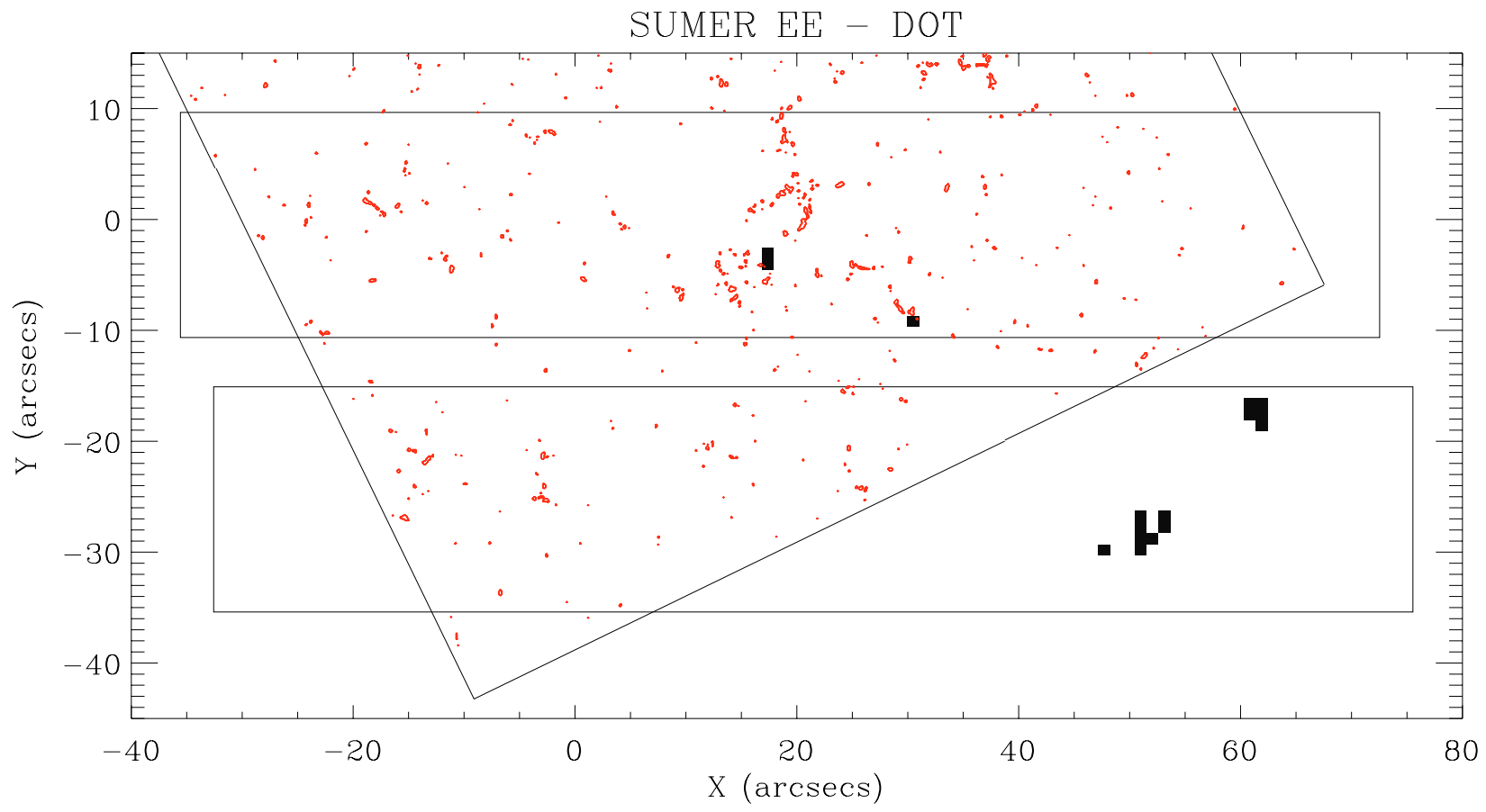

Gband BP 25-Mar-2006 10:59:01.000

Fig. 10. Image of the pixels with EEs (non-Gaussian line profiles) together with the G-band BPs. The EE pixels are represented in black. The rest of the layout remains as for Fig. 2.

down outside the FOV. In this case, however, one would have to understand why the BPs avoid the brightest area. One possibility would be that magnetic structures are present, but they do not show up as BPs because the field strength is too low, the intergranular lanes too cold, etc. (see Sánchez Almeida et al. 2001).
Then the question of why the magnetic structures at this particular location have peculiar properties remains.

The TR loop model by Spadaro et al. (2006) satisfactorily reproduces two non-trivial observables of the quiet Sun TR, namely, the emission measure versus temperature 
distribution, and the temperature dependence of the persistent redshifts. According to this model the spectral lines forming at temperatures similar to that of C III $97.7 \mathrm{~nm}\left(\sim 8 \times 10^{4} \mathrm{~K}\right)$ are systematically redshifted. The corresponding plasma velocities turn out to be in the range between 8 and $15 \mathrm{~km} \mathrm{~s}^{-1}$. We find that the position of the C III $97.7 \mathrm{~nm}$ line profiles measured in the G-band BPs exhibit redshifts with respect to its rest wavelength corresponding to average plasma velocities of $13 \pm 5 \mathrm{~km} \mathrm{~s}^{-1}$ (Fig. 6), in good agreement with the predictions of the TR loop model. Moreover, C III $97.7 \mathrm{~nm}$ spectra appear to be systematically redshifted by about $2 \mathrm{~km} \mathrm{~s}^{-1}$ with respect to the average TR (Sect. 3.2). These results are consistent with the BPs being footpoints of loop-like structures.

Explosive Events (EEs) are believed to be the result of magnetic reconnection. Where the reconnection takes place is under debate. There are authors proposing for reconnection in either the photosphere/chromosphere (the reconnection in the photosphere leads to shocks that accelerate the plasma at TR temperatures, Tarbell et al. 2000), the TR (direct formation of bidirectional jets at the reconnection site, e.g., Innes et al. 1997), and the corona (reconnection high in the corona generates high energy particle beams that heat and accelerate the chromospheric plasma leading to the TR signature, Benz \& Krucker 1999). We have identified several of those events in our FOV, finding a tendency to avoid BPs. This is a new result suggesting that EEs are not located low in the chromosphere (at least not lower than the point where the field starts expanding significantly). If BPs are footpoints of loops undergoing reconnection, then the fact that the EEs do not coincide with them indicates that site of TR plasma acceleration is far from the photospheric footpoints. This would exclude the hypothesis of a flare-like mechanism, as the particle beams reaching the loop footpoints would result in plasma accelerated at, or very close to, the footpoints. In case of shocks formed by reconnection in the photosphere, these shocks travel more than one Mm (distance between the observed EEs and the closest BPs) before being dissipated. Reconnection and consequent heating and plasma acceleration in the low chromosphere seems also excluded. However, it should be mentioned that EEs have a weak signature in chromospheric lines (Teriaca et al. 2002) and that there is some evidence that EEs are first observed in chromospheric lines, and then in TR lines (Madjarska \& Doyle 2002).

As mentioned at the beginning of the section, this work represents a first attempt to outline research avenues for identifying the footpoints of quiet Sun TR loops. Clearly, the temporal and the angular resolution of both the visible and the UV data must be improved to proceed further. Observations obtained with SUMER, DOT and instruments on the satellite Hinode ${ }^{1}$ are expected to yield such improvements.

Acknowledgements. The authors acknowledge the use of the Solar Soft package for data reduction and analysis. This work has been partly funded by the Spanish Ministry of Education and Science (AYA2004-05792), and by the Italian Space Agency (ASI I/035/05/0). The SUMER instrument and its operation are financed by the Deutsches Zentrum für Luft- und Raumfahrt (DLR), the Centre National d'Études Spatiales (CNES), the National Aeronautics and Space Administration (NASA), and the European Space Agency's (ESA) PRODEX programme (Swiss contribution). The instrument is part of ESA's and NASA's Solar and Heliospheric Observatory (SoHO). The DOT is operated by Utrecht University at the Observatorio del Roque de los Muchachos of the IAC.

\section{References}

Athay, R. G. 1982, ApJ, 263, 982

Beckers, J. M. 1977, in Illustrated Glossary for Solar and Solar-Terrestrial Physics, ed. A. Bruzek, \& C. J. Durrant (Dordrecht: Reidel), 21

Benz, A. O., \& Krucker, S. 1999, A\&A, 341, 286

Berger, T. E., Schrijver, C. J., Shine, R. A., et al. 1995, ApJ, 454, 531

Berger, T. E., Löfdahl, M. G., Shine, R. A., \& Title, A. M. 1998, ApJ, 495, 973

Bovelet, B., \& Wiehr, E. 2003, A\&A, 412, 249

Cattaneo, F. 1999, ApJ, 515, L39

de Wijn, A. G., Rutten, R. J., Haverkamp, E. M. W. P., \& Sütterlin, P. 2005, A\&A, 441, 1183

Dere, K. P., Bartoe, J.-D. F., \& Brueckner, G. E. 1989, Sol. Phys., 123, 41

Domínguez Cerdeña, I., Kneer, F., \& Sánchez Almeida, J. 2003, ApJ, 582, L55 Domínguez Cerdeña, I., Sánchez Almeida, J., \& Kneer, F. 2006, ApJ, 636, 496

Dowdy, J. F., Rabin, D., \& Moore, R. L. 1986, Sol. Phys., 105, 35

Faurobert-Scholl, M. 1993, A\&A, 268, 765

Feldman, U. 1983, ApJ, 275, 367

Feldman, U. 2002, in From Solar Min to Max: Half a Solar Cycle with SOHO, ed. A. Wilson (Noordwijk: ESA Publications Division), ESA SP-508, 531

Feldman, U., Widing, K. G., \& Warren, H. P. 1999, ApJ, 522, 1133

Feldman, U., Dammasch, I. E., \& Wilhelm, K. 2000, Space Sci. Rev., 93, 411

Feldman, U., Dammasch, I. E., \& Wilhelm, K. 2001, ApJ, 558, 423

Gabriel, A. H. 1976, Phil. Trans. Roy. Soc. Lond. Series A, 281, 339

Innes, D. E., Inhester, B., Axford, W. I., \& Willhelm, K. 1997, Nature, 386, 811 Jendersie, S., \& Peter, H. 2006, A\&A, 460, 901

Kelly, R. L. 1987, Atomic and ionic spectrum lines below 2000 Angstroms, Hydrogen through Krypton (New York: American Institute of Physics (AIP), American Chemical Society and the National Bureau of Standards)

Lemaire, P., Wilhelm, K., Curdt, W., et al. 1997, Sol. Phys., 170, 105

Lin, H., \& Rimmele, T. 1999, ApJ, 514, 448

Liu, Y., \& Norton, A. A. 2001, MDI measurement errors: the magnetic perspective, Tech. Rep. SOI O1-144, Center for Space Science and Astrophysics, Stanford University

Livingston, W. C., \& Harvey, J. W. 1975, BAAS, 7, 346

Madjarska, M. S., \& Doyle, J. G. 2002, A\&A, 382, 319

Mariska, J. T. 1992, The Solar Transition Region, Cambridge Astrophysics Series (Cambridge: Cambridge University Press)

Martin, B. R. 1971, Statistics for Physicists (London: Academic Press)

Morton, D. C. 2003, ApJS, 149, 205

Muller, R., \& Roudier, T. 1984, Sol. Phys., 94, 33

Pauluhn, A., Solanki, S. K., Rüedi, I., Landi, E., \& Schühle, U. 2000, A\&A, 362, 737

Rutten, R. J., Hammerschlag, R. H., Bettonvil, F. C. M., Sütterlin, P., \& de Wijn, A. G. 2004, A\&A, 413, 1183

Sánchez Almeida, J., \& Lites, B. W. 2000, ApJ, 532, 1215

Sánchez Almeida, J., Asensio Ramos, A., Trujillo Bueno, J., \& Cernicharo, J. 2001, ApJ, 555, 978

Sánchez Almeida, J., Emonet, T., \& Cattaneo, F. 2003, ApJ, 585, 536

Sánchez Almeida, J., Márquez, I., Bonet, J. A., Domínguez Cerdeña, I., \& Muller, R. 2004, ApJ, 609, L91

Scherrer, P. H., Bogart, R. S., Bush, R. I., et al. 1995, Sol. Phys., 162, 129

Schrijver, C. J., \& Title, A. M. 2003, ApJ, 597, L165

Smithson, R. C. 1975, BAAS, 7, 346

Socas-Navarro, H., \& Sánchez Almeida, J. 2002, ApJ, 565, 1323

Spadaro, D., Lanza, A. F., Karpen, J. T., \& Antiochos, S. K. 2006, ApJ, 642, 579

Stein, R. F., \& Nordlund, Å. 2006, ApJ, 642, 1246

Stenflo, J. O. 1982, Sol. Phys., 80, 209

Tarbell, T. D., Ryutova, M., \& Shine, R. 2000, Sol. Phys., 193, 195

Teriaca, L., Banerjee, D., \& Doyle, J. G. 1999, A\&A, 349, 636

Teriaca, L., Madjarska, M. S., \& Doyle, J. G. 2002, A\&A, 392, 309

Teriaca, L., Banerjee, D., Falchi, A., Doyle, J. G., \& Madjarska, M. S. 2004, A\&A, 427, 1065

Title, A. M., \& Berger, T. E. 1996, ApJ, 463, 797

Trujillo Bueno, J., Shchukina, N. G., \& Asensio Ramos, A. 2004, Nature, 430, 326

Vögler, A., \& Schüssler, M. 2007, A\&A, 465, L43

Vögler, A., Shelyag, S., Schüssler, M., et al. 2005, A\&A, 429, 335

Wang, J., Wang, H., Tang, F., Lee, J. W., \& Zirin, H. 1995, Sol. Phys., 160, 277

Warren, H. P., \& Winebarger, A. R. 2000, ApJ, 535, L63

Wilhelm, K., Curdt, W., Marsch, E., et al. 1995, Sol. Phys., 162, 189 